\title{
Crust-core transition of a neutron star: effect of the temperature under strong magnetic fields
}

\author{
Márcio Ferreira ${ }^{1}$, Aziz Rabhi $^{1}$, Constança Providência ${ }^{1}$ \\ CFisUC, Department of Physics, University of Coimbra, 3004-516 Coimbra, Portugal. \\ Received: date / Revised version: date
}

\begin{abstract}
The effect of temperature on the crust-core transition of a magnetar is studied. The thermodynamical spinodals are used to calculate the transition region within a relativistic mean-field approach for the equation of state. Magnetic fields with intensities $5 \times 10^{16} \mathrm{G}$ and $5 \times 10^{17} \mathrm{G}$ are considered. It is shown that the effect on the extension of the crust-core transition is washed away for temperatures above $10^{9} \mathrm{~K}$ for magnetic field intensities $\lesssim 5 \times 10^{16} \mathrm{G}$ but may still persist if a magnetic field as high as $5 \times 10^{17} \mathrm{G}$ is considered. For temperatures below that value, the effect of the magnetic field on crust-core transition is noticeable and grows as the temperature decreases and, in particular, it is interesting to identify the existence of disconnected non-homogenous matter above the $B=0$ crust core transition density. Models with different symmetry energy slopes at saturation show quite different behaviors. In particular, a model with a large slope, as suggested by the recent results of PREX-2, predicts the existence of up to four disconnected regions of non-homogeneous matter above the zero magnetic field crust-core transition density.
\end{abstract}

PACS. XX.XX.XX No PACS code given

\section{Introduction}

Magnetars are considered to be highly magnetized young neutron stars which present a very wide variety of different types of activity, and, in particular, are sources of huge amounts of energy in the form of both electromagnetic and gravitational waves, see [1] for a review. In particular, soft-gamma repeaters (SGRs) and anomalous x-ray pulsars (AXPs) were some of the first magnetars to be identified in astronomy [2,3. These neutron stars have surface magnetic fields as high as $10^{14}-10^{15} \mathrm{G}$. Presently about 30 neutrons stars are known that fall in this category $4^{1}$.

In [5, 6, 7, 8, the authors have discussed, using a dynamical spinodal approach that the extension of the spinodal region of nuclear matter in the space defined by the proton and neutron densities, i.e. $\left(\rho_{p}, \rho_{n}\right)$, is larger than the $B=0$ spinodal region for magnetic field intensities larger than $10^{16} \mathrm{G}$. Moreover, they have predicted from the crossing of a well defined proton fraction curve, as expected inside neutron stars, and the spinodal surface that extra disconnected non-homogeneous regions could exist close but at larger densities than the $B=0$ crust-core transition. To determine the dynamical spinodal, the region where the frequency of density fluctuations goes to zero was calculated. A similar conclusion was drawn un-

Correspondence to: Constança Providência, cp@uc.pt $1 \mathrm{SGR} / \mathrm{APX} \quad$ online catalogue, urlhttp://www.physics.mcgill.ca/ pulsar/magnetar/main.html der a thermodynamical spinodal approach in $[9,10,11,7$, 12. In this approach the instability region corresponds to the region where the curvature of the free energy density is negative. In [13, it has been shown that both approaches give a good prevision of the crust-core transition density inside a neutron star, although the prediction obtained with the thermodynamical spinodal could be approximately $10 \%$ larger.

The main reason for the different behavior of magnetized with respect to non-magnetized nuclear matter is dictated by the Landau quantization of the proton energy due to its charge as discussed in [14]. Besides, including the anomalous magnetic moment (AMM) of both nucleons an extra polarization effect is expected that also affects the properties of nuclear matter in thermodynamic equilibrium [14,15. In the neutron star core very strong magnetic fields are necessary to affect the EoS, see for instance [14,16, 17, while from the virial theorem [18] or from the integration of the Einstein equations coupled to the electromagnetic ones [19,20,21 the strongest fields that a neutron star can support are of the order of $10^{18}$ G. Although, stronger fields are needed to affect the structure of the core EoS, much weaker magnetic fields have a very strong effect on the outer neutron star crust and the onset of the neutron drip as shown in [22,23] (see also 24). Also the inner crust is affected by fields of the order of $10^{17}-10^{18} \mathrm{G}$ as shown in [25,26, and more recently even with weaker fields [27]. In [28], it was shown that the neutral magnetic field line of a poloidal configuration 
could fall inside an extended crust as predicted in [5, 6, 27, which could explain effects as the fracture of the crust.

A neutron star is formed with temperatures above $10^{10}$ $\mathrm{K}$ but within minutes its temperature is below $10^{9} \mathrm{~K}$, and after several kyrs it is expected to have cooled down to surface temperatures below $10^{6} \mathrm{~K}$ and an inner temperature of the order of $10^{8} \mathrm{~K}$ 29]. However, in [30] it was shown that young magnetars with strong surface magnetic fields $\sim 10^{15} \mathrm{G}$ could have surface temperatures magnetars up to $5 \times 10^{6} \mathrm{~K}$ and inner temperatures, at the bottom of the envelope corresponding to a density of the order of $10^{10}-3 \times 10^{11} \mathrm{~g} / \mathrm{cm}^{3}$, up to $\sim 5 \times 10^{8} \mathrm{~K}$. Similar conclusions were drawn in other studies $31,32,33,34$. According to [2,3] the large luminosities of magnetars originate on the huge amounts of magnetic energy those stars store. In 35 the authors have studied several mechanisms, such as mechanical dissipation in the solid crust or heat flux from the liquid core, that convert the magnetic field energy into heat.

However, it is worth mentioning a recent study on the composition of the outer crust of non-accreting neutron star where it has been discussed that the composition of the cristalized crust is not the one determined by the cold catalyzed-matter hypothesis but the one occuring at the crystallization temperature of the crust. This scenario is expected if the NS cooling is fast compared to the nuclear reaction rates that determine cold catalyzed-matter [36, 37. Although in [38] no magnetic field effects were considered, it raises extra questions concerning the composition of the magnetar crust that need to be addressed in the future.

The possibility that magnetars have temperatures in their interior larger than the ones expected inside a neutron star sets the question whether temperature may wash away the effect of Landau quantization, and in particular, the enlarged extension of the crust predicted at $T=0$ $\mathrm{MeV}$. A first study was undertaken in [7, where the effect of temperatures below $1 \mathrm{MeV}$ on the crust properties of the magnetized stars with crust magnetic fields equal to $5 \times 10^{15}-5 \times 10^{16}$ was analysed. It was shown that a temperature of the order of $10 \mathrm{keV}$ would wash away the effect of the enlargement of the crust for fields below $2 \times 10^{16}$ $\mathrm{G}$. In the present study we want to deepen the analysis of the competition between the effect of Landau quantization and temperature on the crust-core transition. However, we only consider temperature effects on the single-particle occupation of excited states above the Fermi surface. Other temperature effects may affect the structure of the crust, in particular, the contribution of the electron-ion interaction to the melting of Coulomb lattice as discussed in 39 , or considering that the pasta phases behave as liquid crystals instead of rigid solids 40 the thermally induced displacements and deformation of the clusters may lead to the melting of the Coulomb lattice at very low temperatures [41,42.

The present paper is organised in the following way: in Sec. we make a brief presentation of the nuclear models that will be applied in the analysis; in Sec. 3 we review the formalism used to calculate the thermodynamic spinodals; in Sec. 4 the results for two magnetic field intensities and several temperatures are presented and discussed and in Sec. 5 some conclusions are drawn.

\section{Nuclear matter model}

In the present section we review the formalism applied to described magnetized stellar matter within a nuclear RMF approach 14,43. We also introduce the anomalous magnetic moment (AMM) and will discuss its effect. We consider the usual Lagrangian density

$$
\mathcal{L}=\sum_{i=p, n} \mathcal{L}_{i}+\mathcal{L}_{e}+\mathcal{L}_{\sigma}+\mathcal{L}_{\omega}+\mathcal{L}_{\rho}+\mathcal{L}_{\omega \rho}
$$

where the different terms are: $\mathcal{L}_{i}$ the nucleon Lagrangian density, given by

$$
\mathcal{L}_{i}=\bar{\psi}_{i}\left[\gamma_{\mu} i D^{\mu}-M_{i}^{*}-\frac{1}{2} \mu_{N} \kappa_{i} \sigma_{\mu \nu} F^{\mu \nu}\right] \psi_{i},
$$

with

$$
i D^{\mu}=i \partial^{\mu}-g_{v} V^{\mu}-\frac{g_{\rho}}{2} \boldsymbol{\tau} \cdot \mathbf{b}^{\mu}-e A^{\mu} \frac{1+\tau_{3}}{2},
$$

and $M_{p}^{*}=M_{n}^{*}=M^{*}=m-g_{s} \phi$; the mesonic terms for the $\sigma, \omega$ and $\rho$-mesons, $\mathcal{L}_{\sigma}, \mathcal{L}_{\omega}$ and $\mathcal{L}_{\rho}$, are defined as in [6] in terms of the fields $\phi, V^{\mu}$ and $\boldsymbol{b}^{\mu}$ with masses $m_{\sigma}$, $m_{v}$ and $m_{\rho}$, respectively,

$$
\begin{aligned}
& \mathcal{L}_{\sigma}=\frac{1}{2}\left(\partial_{\mu} \phi \partial^{\mu} \phi-m_{\sigma}^{2} \phi^{2}-\frac{1}{3} \kappa \phi^{3}-\frac{1}{12} \lambda \phi^{4}\right), \\
& \mathcal{L}_{\omega}=-\frac{1}{4} \Omega_{\mu \nu} \Omega^{\mu \nu}+\frac{1}{2} m_{v}^{2} V_{\mu} V^{\mu}+\frac{1}{4 !} \xi g_{v}^{4}\left(V_{\mu} V^{\mu}\right)^{2}, \\
& \mathcal{L}_{\rho}=-\frac{1}{4} \mathbf{B}_{\mu \nu} \cdot \mathbf{B}^{\mu \nu}+\frac{1}{2} m_{\rho}^{2} \mathbf{b}_{\mu} \cdot \mathbf{b}^{\mu},
\end{aligned}
$$

where $\Omega_{\mu \nu}=\partial_{\mu} V_{\nu}-\partial_{\nu} V_{\mu}$, and $\mathbf{B}_{\mu \nu}=\partial_{\mu} \mathbf{b}_{\nu}-\partial_{\nu} \mathbf{b}_{\mu}-$ $g_{\rho}\left(\mathbf{b}_{\mu} \times \mathbf{b}_{\nu}\right)$, and the parameters $\kappa$ and $\lambda$ are associated with the third- and fourth-order terms of the scalar field. The term

$$
\mathcal{L}_{\omega \rho}=\Lambda_{v} g_{v}^{2} g_{\rho}^{2} V_{\mu} V^{\mu} \mathbf{b}_{\mu} \cdot \mathbf{b}^{\mu},
$$

couples the $\rho$ and the $\omega$ meson. Changing the strength of this term will allow to make the symmetry energy softer (harder) increasing (decreasing) the coupling [44, 45, 46]. In our study we consider the models TM1 47] and TM1e [48. Both parametrizations describe symmetric nuclear matter with the same functional and only differ in the isovector channel. Both describe two solar mass stars 49 , 48,50. Finally, $\mathcal{L}_{e}$ is the electron Lagrangian density, Electrons, with mass $m_{e}$, are also included in the Lagrangian density,

$$
\mathcal{L}_{e}=\bar{\psi}_{e}\left[i \gamma_{\mu} \partial^{\mu}-m_{e}\right] \psi_{e}
$$

when the $\beta$-equilibrium EoS is calculated. Since we will only discuss the crust-core transition, muons will not be included as they set in at $\rho \gtrsim 0.11 \mathrm{fm}^{-3}$ above the crustcore transition. 
Protons interact with a static field $A^{\mu}$, which includes a static component assumed to be externally generated, $A^{\mu}=(0,0, B x, 0)$, so that $\mathbf{B}=B \hat{z}$ and $\nabla \cdot \mathbf{A}=0$. The nucleon AMM is introduced via the coupling of the baryons to the electromagnetic field tensor with $\sigma_{\mu \nu}=\frac{i}{2}\left[\gamma_{\mu}, \gamma_{\nu}\right]$, and strength $\kappa_{i}$, with $\kappa_{n}=-1.91315$ for the neutron, and $\kappa_{p}=1.79285$ for the proton. $\mu_{N}$ is the nuclear magneton.

The properties of TM1 and TM1e models at saturation [50] are the same for the isoscalar channel, in particular, the binding energy $E_{b}=-16.3 \mathrm{MeV}$, the saturation density $\rho_{0}=0.145 \mathrm{fm}^{-3}$, and the incompressibility $K=281$ $\mathrm{MeV}$. The two models differ in the isovector properties: the symmetry energy $J$ and its slope $L$ at saturation, take, respectively, the values $36.89 \mathrm{MeV}$ and $110.8 \mathrm{MeV}$ for TM1 and $31.38 \mathrm{MeV}$ and $40 \mathrm{MeV}$ for TM1e. The last model satisfies the constraints set by microscopic calculations of neutron matter in a chiral effective field approach [51. On the other hand, recent results from the Lead Radius EXperiment (PREX-2) collaboration have extracted from the measurements of the parity-violating asymmetry a neutron skin thickness $R_{n}-R_{p}=0.283 \pm 0.071 \mathrm{fm}[52$, which, according to [53, corresponds to a slope of the symmetry energy at saturation in the range $L=106 \pm 37$ $\mathrm{MeV}$, taking into account the strong correlation existing between the neutron skin thickness and the symmetry energy slope $L$. These results justify that we also consider model TM1 with larger symmetry energy than TM1e, well inside the range proposed in 53 .

\section{Thermodynamical spinodal}

In the present section we introduce the thermodynamical spinodal which will be used to estimate the transition from a clusterized to homogeneous region. In particular, we will show how the spinodal changes with temperature and how temperature affects the transition region.

The state which minimizes the free energy of asymmetric npe matter is characterized by the nucleon distribution functions

$$
f_{i \pm}=\frac{1}{1+e^{\left(\epsilon_{i} \mp \nu_{i}\right) / T}} \quad i=p, n
$$

where the effective chemical potential $\nu_{i}$ is related to the chemical potential $\mu_{i}$ by the equation

$$
\nu_{i}=\mu_{i}-g_{v} V_{0}-\frac{g_{\rho}}{2} \tau_{i} b_{0}
$$

and the electron distribution function

$$
f_{e \pm}=\frac{1}{1+e^{\left(\epsilon_{e} \mp \mu_{e}\right) / T}},
$$

with $\mu_{e}$ the electron chemical potential. In these expressions the quasiparticle energies are given by

$$
\begin{aligned}
\epsilon_{p} & =\sqrt{p_{z}^{2}+\bar{m}_{p}^{2}}, \\
\epsilon_{n} & =\sqrt{p_{z}^{2}+\left(\sqrt{M^{* 2}+p_{\perp}^{2}}-s \mu_{N} \kappa_{n} B\right)^{2}}, \\
\epsilon_{e} & =\sqrt{p_{z}^{2}+\bar{m}_{e}^{2}} .
\end{aligned}
$$

where

$$
\bar{m}_{p}=\tilde{m}_{p, \nu}-s \mu_{N} \kappa_{p} B \equiv \bar{m}_{p, \nu s}
$$

with $\tilde{m}_{p, \nu}=\sqrt{M^{* 2}+2 \nu q_{p} B}, \bar{m}_{e}=\sqrt{m_{e}+2 \nu e B} \equiv \bar{m}_{e, \nu}$, $\nu$ designates the Landau level and $s= \pm 1$ is the spin projection in the direction of the magnetic field.

In the above expressions the constant mesonic fields satisfy the following equations

$$
\begin{aligned}
m_{\sigma}^{2} \phi_{0}+\frac{\kappa}{2} \phi_{0}^{2} & +\frac{\lambda}{6} \phi_{0}^{3}=g_{s}\left(\rho_{s p}+\rho_{s n}\right), \\
m_{v}^{2} V_{0} & =g_{v}\left(\rho_{p}+\rho_{n}\right), \\
m_{\rho}^{2} b_{0} & =\frac{g_{\rho}}{2}\left(\rho_{p}-\rho_{n}\right),
\end{aligned}
$$

the spatial components of the vector mesons being zero since we consider a static description. At zero temperature the above distribution functions reduce to $f_{i+}=\theta\left(P_{F i}^{2}-\right.$ $p^{2}$ ) where $P_{F i}$ is the Fermi momentum of nucleon $i$ and $f_{i-}=0$ [54,55].

In terms of the finite temperature distribution functions the scalar and vector densities are given by

$$
\rho_{s p}=\frac{e B}{(2 \pi)^{2}} \sum_{\nu, s} \int d p_{z}\left(f_{p+}+f_{p-}\right) \frac{\bar{m}_{p} M^{*}}{\left(\bar{m}_{p}+s \mu_{N} \kappa_{p} B\right) \epsilon_{p}}
$$

$$
\rho_{s n}=\sum_{s} \int \frac{d^{3} p}{(2 \pi)^{3}}\left(f_{n+}+f_{n-}\right)\left[1-\frac{s \mu_{N} \kappa_{n} B}{\sqrt{M^{* 2}+p_{\perp}^{2}}}\right] \frac{M^{*}}{\epsilon_{n}}
$$

$$
\begin{gathered}
\rho_{p}=\frac{e B}{(2 \pi)^{2}} \sum_{\nu, s} \int d p_{z}\left(f_{p+}-f_{p-}\right), \\
\rho_{n}=\sum_{s} \int \frac{d^{3} p}{(2 \pi)^{3}}\left(f_{n+}-f_{n-}\right), \\
\rho_{e}=\frac{e B}{(2 \pi)^{2}} \sum_{\nu, s} \int d p_{z}\left(f_{e+}-f_{e-}\right) .
\end{gathered}
$$

The stability of nuclear matter is determined imposing that the free energy density $\mathcal{F}$ is a convex function of the densities $\rho_{p}$ and $\rho_{n}$. For stable homogeneous matter, the symmetric curvature matrix with the elements [56, 57, 58],

$$
\mathcal{F}_{i j}=\left(\frac{\partial^{2} \mathcal{F}}{\partial \rho_{i} \partial \rho_{j}}\right)_{T}
$$

must be positive. This is equivalent to imposing that the trace and the determinant of $\mathcal{F}_{i j}$ are positive. In terms of the proton and neutron chemical potentials $\mu_{i}$, the curvature matrix is given by

$$
\mathcal{F}=\left(\begin{array}{ll}
\frac{\partial \mu_{n}}{\partial \rho_{n}} & \frac{\partial \mu_{n}}{\partial \rho_{p}} \\
\frac{\partial \mu_{p}}{\partial \rho_{n}} & \frac{\partial \mu_{p}}{\partial \rho_{p}}
\end{array}\right)
$$


with $\mu_{i}=\left.\frac{\partial \mathcal{F}}{\partial \rho_{i}}\right|_{T, \rho_{j \neq i}}$. For nuclear matter, the largest eigenvalue of the curvature matrix is always positive and the other becomes negative at sub-saturation densities. The surface characterized by a zero determinant of $\mathcal{F}_{i j}$, e.g. a zero eigenvalue, defines the thermodynamical spinodal on the $\left(\rho_{n}, \rho_{p}, T\right)$ space. Inside the thermodynamical spinodal the smallest eigenvalue of $\mathcal{F}_{i j}$ nuclear matter is negative corresponding to an unstable state. The curvature matrix has the eigenvalues

$$
\lambda_{ \pm}=\frac{1}{2}\left(\operatorname{Tr}(\mathcal{F}) \pm \sqrt{\operatorname{Tr}(\mathcal{F})^{2}-4 \operatorname{Det}(\mathcal{F})}\right) .
$$

According to the stability condition both must be positive. A thermodynamic instablility is obtained if one becomes negative. In this case, the system will decrease its free energy evolving in the instability direction, which is the direction of the eigenvector associated to the negative eigenvalue. The eigenvectors $\delta \rho_{ \pm}$are given by

$$
\frac{\delta \rho_{i}^{ \pm}}{\delta \rho_{j}^{ \pm}}=\frac{\lambda_{ \pm}-\mathcal{F}_{j j}}{\mathcal{F}_{j i}}, i, j=p, n .
$$

The free energy density is given by

$$
\mathcal{F}=\varepsilon-T \mathcal{S}
$$

where $T$ is the temperature and $\varepsilon$ is the energy density

$$
\begin{aligned}
\varepsilon= & \sum_{i=p, n} \varepsilon_{i}+\frac{1}{2} m_{\sigma}^{2} \phi^{2}+\frac{1}{3} \kappa \phi^{3}+\frac{1}{12} \lambda \phi^{4} \\
& +\frac{1}{2} m_{v}^{2} V_{0}^{2}+\frac{1}{2} m_{\rho}^{2} b_{0}^{2}+\frac{1}{8} \xi g_{v}^{4} V_{0}^{4}+3 \Lambda_{v} g_{v}^{2} g_{\rho}^{2} V_{0}^{2} b_{0}^{2},
\end{aligned}
$$

and $\mathcal{S}=\mathcal{S}_{p}+\mathcal{S}_{n}$ is the entropy density. In the above expressions the proton energy density $\varepsilon_{p}$ and the neutron energy density $\varepsilon_{n}$ are defined by [4]

$$
\begin{aligned}
& \varepsilon_{p}=\frac{e B}{2 \pi^{2}} \sum_{\nu, s} \int_{0}^{\infty} d p_{z} \epsilon_{p}\left(f_{p+}+f_{n-}\right), \\
& \varepsilon_{n}=\sum_{s} \int \frac{d^{3} p}{(2 \pi)^{3}} \epsilon_{n}\left(f_{n+}+f_{n-}\right)
\end{aligned}
$$

and the proton and neutron entropy densities by

$$
\begin{aligned}
\mathcal{S}_{p}= & -\frac{e B}{2 \pi^{2}} \sum_{\nu, s} \int_{0}^{\infty} d p_{z}\left\{f_{p+} \ln f_{p+}\right. \\
& \left.+\left(1-f_{p+}\right) \ln \left(1-f_{p+}\right)+(p+\rightarrow p-)\right\} \\
\mathcal{S}_{n}= & -\sum_{s} \int \frac{d p^{3}}{(2 \pi)^{3}}\left\{f_{n+} \log f_{n+}\right. \\
& \left.+\left(1-f_{n+}\right) \log \left(1-f_{n+}\right)+(n+\rightarrow n-)\right\} .
\end{aligned}
$$

In order to calculate the crust-core transition, we will determine the crossing of the stellar matter equation of state with the spinodal surface. Two different scenarios will be considered: i) $\beta$-equilibrium neutrino free cold and warm matter; ii) matter with a charge fraction $Y_{p}=0.1$ a fraction that is larger than the one of $\beta$-equilibrium and which occurs in the proto-neutron star already after the diffusion of neutrinos out of the star. The crust-core transition will be determined from the crossing of the $\beta$ equilibrium proton fraction, and the $Y_{p}=0.1$ curve with the spinodal section. For $T=0$, it was shown that there may exist a sequence of unstable bands separated by stable bands due to the existence of Landau levels. However, the effects Landau quantization are diluted by the inclusion of temperature and this effect will be discussed in the following.

\section{Results}

In the present section, we will show some calculations of the spinodal surface performed for two reference magnetic field intensities: $B^{*}=10^{3}$ and $10^{4}$ corresponding to $4.4 \times 10^{16} \mathrm{G}$ and $4.4 \times 10^{17} \mathrm{G}$ since $B^{*}=B / B_{c e}$ with $B_{c e}=4.4 \times 10^{13} \mathrm{G}$ the critical electron field. These intensities could occur in the interior of a magnetar $[59,60$, 61 . In 60,61, and contrary to 59, both a poloidal and toroidal component are considered, and the localization of the toroidal lines of fields with a magnitude that can be as high as $B^{*}=10^{3}$ to $10^{4}$ close to the surface, where one may expect the crust-core transition to occur. It should, however, be pointed out that in 61 taking a single segment polytropic EOS, the huge fields were considered, and it was shown that they affect the distribution of matter and create a magnetovacuum region. This indicates that further studies that couple the magnetic field to matter are required.

The magnetized matter will be calculated within the TM1 and TM1e models [47,48] as referred before. These are models that differ in the density dependence of the symmetry energy. We will consider the temperatures $T=$ $10,50,100$ and $500 \mathrm{keV}$. In the low magnetic field limit, we expect the energy difference between Landau levels of the order $\Delta \epsilon \lesssim e B / M^{*}=m_{e}^{2} B^{*} / M^{*} \approx 3 \times 10^{-4} B^{*}$. Concerning the AMM it is good to have as reference that the sum of the proton and neutron contributions for the same spin projection is $\left|\kappa_{p}+\kappa_{n}\right| \mu_{N} B \approx 2 \times 10^{-5} B^{*} \mathrm{MeV}$. For $B^{*}=10^{3}$, its effect is only seen for the smallest temperature we consider, and for $B^{*}=10^{4}$ its effect is washed out for $T \gtrsim 100 \mathrm{keV}$. Due to the computational effort we will only discuss the effect of AMM in one example when its effect is still clearly seen.

In Fig. 1 we represent, for the TM1e model, the region in the $\rho_{p}-\rho_{n}$ space where the eigenvector $\lambda_{-}$defined in Eq. (11) is negative for matter under the effect of a magnetic field intensity $B^{*}=10^{3}$ (left panels) and $B^{*}=$ $10^{4}$ (right panels). The colors indicate the magnitude of the eigenvector and the lines represent the $Y_{p}=0.1$ line (top) and the $\beta$-equilibrium line (bottom). It is clearly seen the structure created by the Landau quantization of the proton energies, which divides the spinodal region into bands which extend well into regions that are stable for $B=0$. Both sections were obtained without the inclusion of the anomalous magnetic field (AMM). The main effect of including the AMM is to divide each band into two narrower bands that are associated with a different spin 

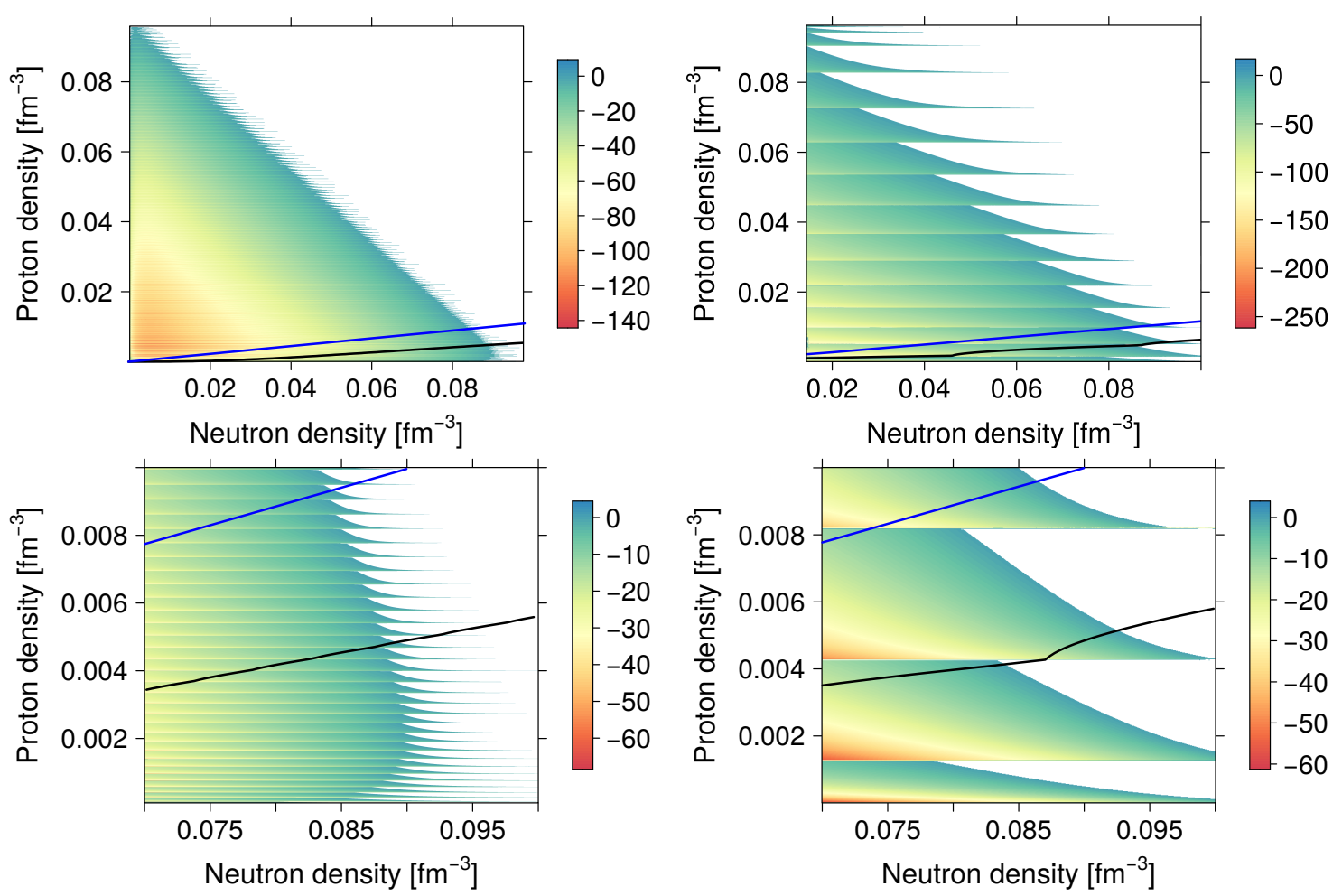

Fig. 1. Spinodal section obtained within the TM1e model for $B^{*}=10^{3}$ (left) and $B^{*}=10^{4}$ (right) at zero temperature. The bottom panels contain a detail at the region of interest for the crust-core transition. No AMM has been considered. The colours show the magnitude of $\lambda_{-}$(see Eq. 11 in units of $(\hbar c)^{3} / M^{2}$. The two lines represented define the $Y_{p}=0.1$ (top) and $\beta$-equilibrium (bottom) proton fractions.
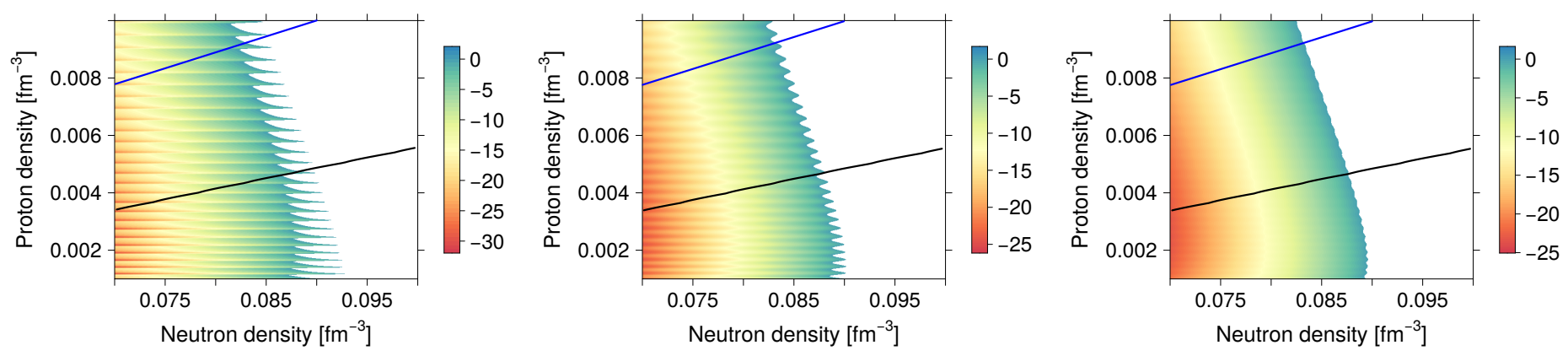

Fig. 2. Spinodal sections obtained within the TM1e model for $B^{*}=10^{3}$ and temperatures $[\mathrm{keV}]: 10$ (left), 50 (center), and 100 (right). The colours show the magnitude of $\lambda_{-}$(see Eq. $\sqrt{11}$ in units of $(\hbar c)^{3} / M^{2}$. The two lines represented define the $Y_{p}=0.1$ (top) and $\beta$-equilibrium (bottom) proton fractions.

projection with respect to the magnetic field direction. The two bottom panels show details for a proton density below $0.01 \mathrm{fm}^{-3}$. This allows to study the transition to non-homogeneous matter with a temperature below 0.5 $\mathrm{MeV}$, in particular, considering a proton fraction below 0.1 , as in protoneutron stars in a neutrino free stage, and temperatures below $1 \mathrm{MeV}$. The extension of the spinodal bands beyond the $B=0$ curve occurs with quite shallow minima and, as will be shown below, the temperature will dissolve these regions. For $T=0$ the $\beta$-equilibrium curves cross several bands so that between the density when the curve first crosses the spinodal surface and the density the curve definitely enters the core, the $\beta$-equilibrium curve has passed through two unstable regions. Taking the curve $Y_{p}=0.1$ three disconnected unstable regions would occur before the onset of the core.

For the largest field, $B^{*}=10^{4}$, it is clearly seen that the opening of a new Landau level occurs at defined values of the proton density and that the larger the proton density, the larger the widths of the bands. For this field both proton fractions represented would give rise to two disconnected unstable regions below the core.

We next study the effect of temperature on the spinodal section of the TM1e model for matter under the effect of a magnetic field with intensity $B^{*}=10^{3}$, see Figs. 2 where the spinodal was calculated for $T=10 \mathrm{keV}$ (left) 

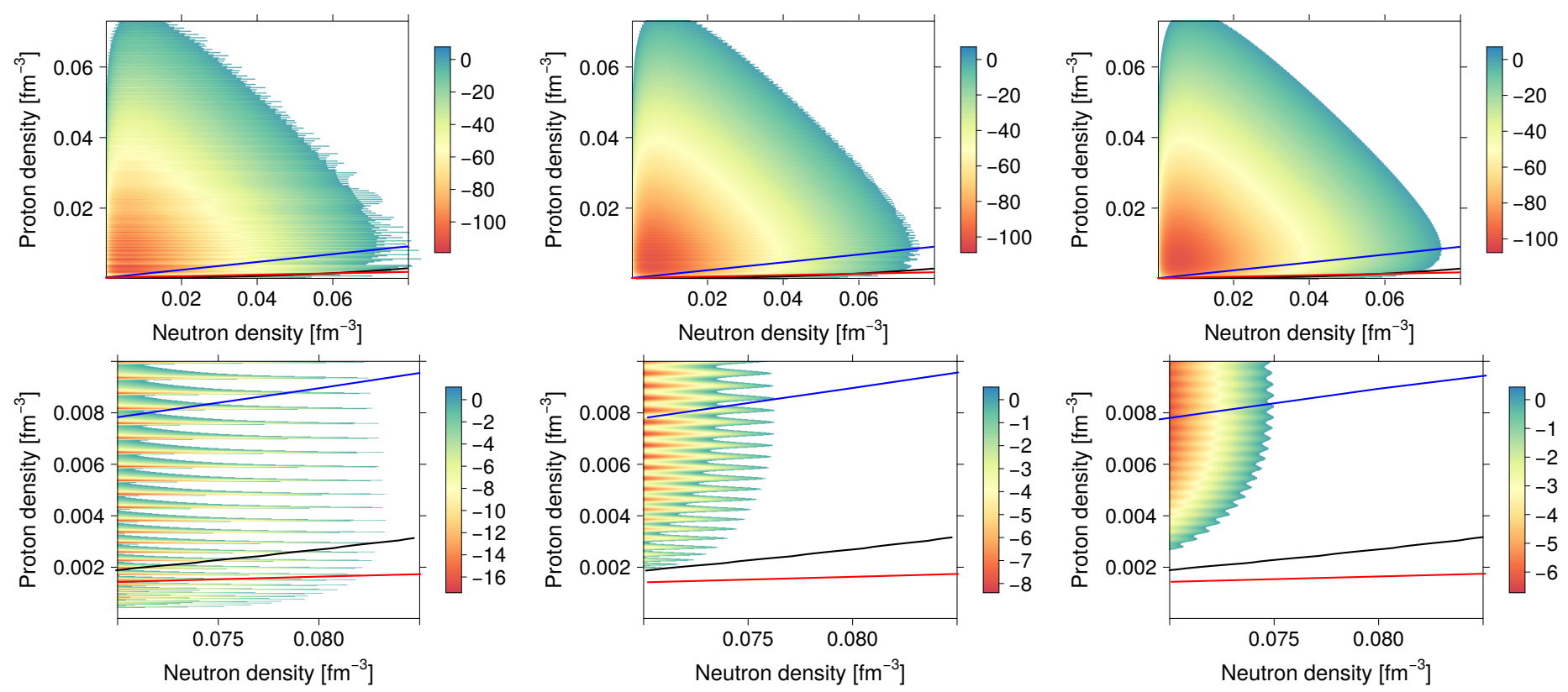

Fig. 3. Spinodal sections obtained within the TM1 model for $B^{*}=10^{3}$ and temperatures [keV]: 10 (left), 50 (center), and 100 (right). The bottom panels contain a detail at the region of interest for the crust-core transition. The colours show the magnitude of $\lambda_{-}$(see Eq. 11 in units of $(\hbar c)^{3} / M^{2}$. The three lines represented define the $Y_{p}=0.1$ (top) and $\beta$-equilibrium (middle) and $Y_{p}=0.02$ (bottom) proton fractions.
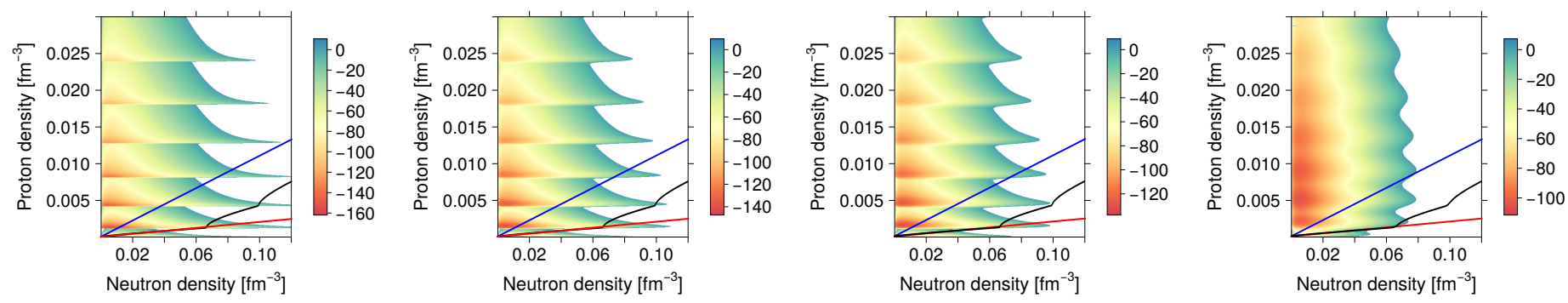

Fig. 4. Spinodal sections obtained within the TM1 model for $B^{*}=10^{4}$ and temperatures [keV]: 10, 50, 100, and 500 (from left to right). The colours show the magnitude of $\lambda_{-}$(see Eq. $\sqrt{11}$ in units of $(\hbar c)^{3} / M^{2}$. The colours show the magnitude of $\lambda_{-}$(see Eq. 11 in units of $(\hbar c)^{3} / M^{2}$. The three lines represented define the $Y_{p}=0.1$ (top) and $\beta$-equilibrium (middle) and $Y_{p}=0.02$ (bottom) proton fractions.

$50 \mathrm{keV}$ (middle) and $100 \mathrm{keV}$ (right) and compare with Fig. 1 left panel calculated for $T=0$. Only the detail of the spinodal necessary to determine the crust-core transition is shown. For $T=10 \mathrm{keV}$ the structure of bands is still clearly seen in the interior of the spinodal surface but the extension of the bands to regions beyond the $B=0$ spinodal surface is now much smaller: from the $T=0$ more than $0.01 \mathrm{fm}^{-3}$ length to less than 0.005 $\mathrm{fm}^{-3}$. This could be expected since the $\lambda_{-}$although negative took values very close to zero at the extreme ends of the bands. At $T=50 \mathrm{keV}$ the band effect is much weaker and at $T=100 \mathrm{keV}$ the interior band structure has completely disappeared but the spinodal surface still shows a slight magnetic field effect. This is expectable since the energy interval between two Landau levels is of the order of $\lesssim e B / M^{*}$, where the approximate refers to the low magnetic field intensities. For $B^{*}=10^{3}$, we have $e B / M^{*} \approx 0.5$ $\mathrm{MeV}$. The two curves shown define the $Y_{p}=0.01$ (top) $\beta$-equilibrium (bottom) proton fractions. The crossing of these curves with the spinodal surface indicate that only for the $\beta$-equilibrium proton fraction two disconnected non-homogeneous regions would appear. Nonetheless, for the two lowest temperatures $T=10$ and $50 \mathrm{keV}$ the crustcore transition still occurs at a larger density than predicted for $B=0$. These three figures also allow to point out a further detail concerning the structure of unstable region: it is clearly seen for $T=100 \mathrm{keV}$ that once the band structure disappears the eigenvalue $\lambda_{-}$is more negative the smaller the neutron density, this is not the case for the other two cases, and for the $T=0$ spinodals shown in Fig. 11 the bands correspond to more negative values of $\lambda_{-}$at the bottom of the band when the Landau level starts being filled and much less at the top of the band.

A similar effect is observed for the TM1 model in Figs. 3. while at $T=10$ and $50 \mathrm{keV}$ the band structure is clearly seen, the effect of the magnetic field has been washed out 

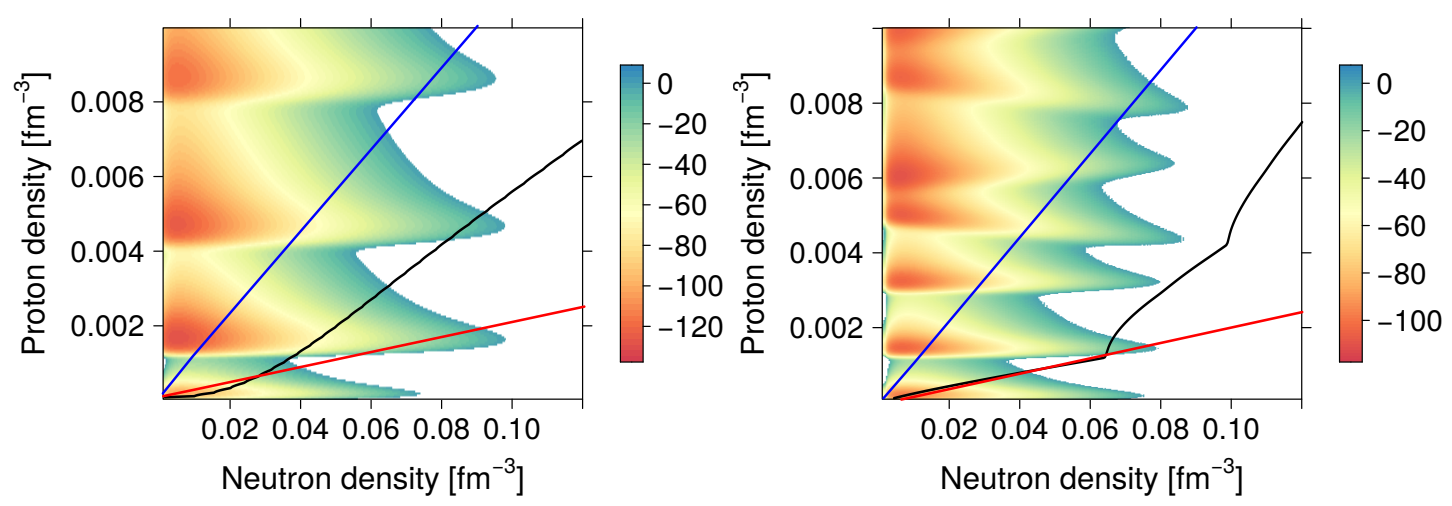

Fig. 5. Spinodal section obtained within the TM1 model for $B^{*}=10^{4}$ and $T=100 \mathrm{keV}$ without (left) and with (right) AMM. The colours show the magnitude of $\lambda_{-}$(see Eq. 11) in units of $(\hbar c)^{3} / M^{2}$. The three lines represented define the $Y_{p}=0.1$ (top) and $\beta$-equilibrium (middle) and $Y_{p}=0.02$ (bottom) proton fractions.

for $T=100 \mathrm{keV}$, except for a small effect at the surface. Comparing with TM1e, the TM1 spinodal surface extends to smaller densities. As already discussed in other works, see for instance 62], this is related to the density dependence of the symmetry energy. However, the extension of the spinodal bands due to Landau quantization beyond the $B=0$ surface is larger as discussed in [6, 7] where different models with similar properties were discussed. This is due to the fact that at low densities the symmetry energy of this model is smaller and allows that asymmetric nuclear matter is more bound than matter described by TM1e. Note, however, that the eigenvalue $\lambda_{-}$takes values very close to zero, and, therefore, temperature dissolves easily these structures. At $T=10 \mathrm{keV}$ the $\beta$-equilibrium curve defines five non-homogeneous disconnected regions while the $Y_{p}=0.1$ curve defines three. The $Y_{p}=0.02$ curve deviates from the $\beta$-equilibrium curve at the $B=0$ transition density and under-estimates the proton fraction. However, the qualitative behavior predicted is similar to the one by the $\beta$-equilibrium curve. At $T=100 \mathrm{keV}$, there are no disconnected unstable regions for all scenarios and for $T=50 \mathrm{keV}$, there is at most one disconnected region. We conclude that within TM1, the crust structure is very sensitive to the temperature evolution, more than within TM1e.

In the following we discuss the effect of a $B^{*}=10^{4}$ magnetic field on the crust-core transition of matter described within the TM1 model. Large fields similar to this one have been reported in studies which include besides a possible poloidal field also a toroidal field [59,63. 61. The sections of the spinodal surfaces of interest to study neutron rich matter are represented in Fig. 4 for $T=10,50,100$ and $500 \mathrm{keV}$. For this strong field even the largest temperature we consider is not enough to completely wash out the band structure, although a much weaker effect is obtained for $T=500 \mathrm{keV}$. For the three smallest temperatures the band structure is similar and only the extension of the bands for larger neutron fractions is affected with smaller densities being attained with larger temperatures. This has implications in the structure of the crust: for $T=10 \mathrm{keV}$ we identify for $\beta$-equilibrium matter at least three disconnected non-homogeneous re- gions; for $T=50 \mathrm{keV}$ we still have three disconnected regions, but this number reduces to two or even only one increasing the temperature to 100 or $500 \mathrm{keV}$.

In Fig. 5 the effect of the AMM is clearly shown in a scenario when it is not negligible: a magnetic field with an intensity $B^{*}=10^{4}$ is considered and a temperature equal to $100 \mathrm{keV}$. For such a strong magnetic field, the temperature $T=100 \mathrm{keV}$ does not remove the effect of Landau quantization. As discussed before, this temperature just reduces the extension of the spinodal bands with respect to colder scenarios. The introduction of AMM has as two main effects: i) the splitting of the spinodal bands into two due to spin polarization. The first band does not suffer any splitting because it is defined by a single spin polarization, but all the other bands have been splitted into two, the separation being larger for the lower bands; ii) the reduction of the extension of the spinodal bands. Being the effect of the AMM one order of magnitude smaller than the effect of Landau quantization, its effect washes away for temperatures that are approximately one order of magnitude smaller.

We have included three curves that describe from top to bottom matter with a proton fraction $Y_{p}=0.1$, matter in $\beta$-equilibrium ad matter with a proton fraction $Y_{p}=0.02$. This last fraction was considered because it represents the proton fraction of the non-homogeneous inner crust matter close to the crust-core transition for $B=0$. This value has been considered in previous studies as in 5 , 6. For the three scenarios described, the crust-core transition is identified with the crossing of the proton fraction curves with the spinodal surface. Some conclusions are in order: i) For $\beta$-equilibrium matter there exists a first crossing from unstable to stable matter around 0.06 $\mathrm{fm}^{-3}$, however, for $\rho \approx 0.08 \mathrm{fm}^{-3}$ the curve crosses again an unstable region before entering definitely the core. Under these conditions the star would have two disconnected non-homogeneous regions, as already predicted in [5, 6]. The qualitative conclusion is not different from the one drawn with $Y_{p}=0.02$; ii) a similar situation will occur also for a scenario when $\beta$-equilibrium was still not attained and the proton fraction is 0.1 , although the crossings occur at different densities. If the AMM is taken into 
account we still get two disconnected non-homogeneous regions for $\beta$-equilibrium matter and not for $Y_{p}=0.1$ because the extension of the bands is smaller. If a larger temperature had been chosen the effect of the AMM would have mostly disappeared.

\section{Conclusions}

We have discussed the effect of temperature on the spinodal surface of magnetized nuclear matter within two RMF models describing symmetric nuclear matter with the same underlying model, however having different density dependences of the symmetry energy. The two models considered, TM1 47] and TM1e [4], satisfy, respectively, the constraints imposed by the recent measurement of PREX2 and the neutron matter constraints obtained within a chiral effective field theoretical calculation 51. In addition we have briefly commented on the effect of including explicitly the nucleon AMM. The magnitude of its effect is approximately one order of magnitude smaller than the effect due to the Landau quantization of the proton energies, and therefore, temperature washes away its effect at lower temperatures. The main effect of the nucleon AMM is to split into two each band in the spinodal region except for the first one, and to slightly reduce the extension of the bands beyond the $B=0$ crust-core transition density. This splitting may define extra disconnected regions but only in the $T=0$ limit.

From the knowledge of the spinodals surfaces it was possible to make a crust-core prevision for different proton fraction scenarios and, in particular, to discuss the possible existence of disconnected non-homogeneous regions and how temperature removes this structure. The main conclusion was that the prevision of a finite number of disconnected unstable regions obtained with $T=0 \mathrm{MeV}$ would reduce to a single one for 100-500 keV depending on the field intensity. This implies that during the cooling or heating of the star, solid regions will form in the interior close to the crust-core transition. Besides, it was shown that this behavior may be more dramatic if the model that describes the nuclear matter has a large symmetry energy slope at saturation density, implying a small symmetry energy at subsaturation densities. In this case it was shown that many disconnected unstable regions would appear at zero temperature. However, the free energy density curvature matrix eigenvalue that defines the instability takes values very close to zero, and, therefore, temperatures easily melts these structures. The complexity of this region and its evolution with cooling may create conditions for a plastic-flow scenario under very strong magnetic fields and allow for a field evolution that generates a twisted corona, as suggested in 64,65$]$.

\section{Acknowledgments}

This work was partially supported by national funds from FCT (Fundação para a Ciência e a Tecnologia, I.P, Portugal) under the Projects No. UID/FIS/04564/2019, No.
UID/04564/2020, and No. POCI-01-0145-FEDER-029912 with financial support from Science, Technology and Innovation, in its FEDER component, and by the FCT/MCTES budget through national funds (OE).

\section{References}

1. V.M. Kaspi, A. Beloborodov, Ann. Rev. Astron. Astrophys. 55, 261 (2017). DOI 10.1146/ annurev-astro-081915-023329

2. R.C. Duncan, C. Thompson, Astrophys. J. Lett. 392, L9 (1992). DOI 10.1086/186413

3. B. Paczynski, Acta Astron. 42, 145 (1992)

4. S.A. Olausen, V.M. Kaspi, Astrophys. J. Suppl. 212, 6 (2014). DOI 10.1088/0067-0049/212/1/6

5. J. Fang, H. Pais, S. Avancini, C. Providência, Physical Review C 94(6) (2016)

6. J. Fang, H. Pais, S. Pratapsi, S. Avancini, J. Li, C. Providência, Physical Review C 95(4) (2017)

7. J. Fang, H. Pais, S. Pratapsi, C. Providência, Phys. Rev. C95(6), 062801 (2017)

8. S. Avancini, B.P. Bertolino, A. Rabhi, J. Fang, H. Pais, C. Providência, Phys. Rev. C 98(2), 025805 (2018). DOI 10.1103/PhysRevC.98.025805

9. A. Rabhi, C. Providencia, J. Da Providencia, Phys. Rev. C 79, 015804 (2009). DOI 10.1103/PhysRevC.79.015804

10. A. Rabhi, C. Providencia, J. Da Providencia, Phys. Rev. C 80, 025806 (2009). DOI 10.1103/PhysRevC.80.025806

11. Y.J. Chen, Phys. Rev. C 95(3), 035807 (2017). DOI 10. 1103/PhysRevC.95.035807

12. D. Chatterjee, F. Gulminelli, D.P. Menezes, JCAP 03, 035 (2019). DOI 10.1088/1475-7516/2019/03/035

13. S.S. Avancini, S. Chiacchiera, D.P. Menezes, C. Providencia, Phys. Rev. C 82, 055807 (2010). DOI 10.1103/ PhysRevC.82.055807. [Erratum: Phys.Rev.C 85, 059904 (2012)]

14. A. Broderick, M. Prakash, J.M. Lattimer, The Astrophysical Journal 537(1), 351 (2000)

15. M.A. Perez-Garcia, C. Providencia, A. Rabhi, Phys. Rev. C 84, 045803 (2011). DOI 10.1103/PhysRevC.84.045803

16. A.E. Broderick, M. Prakash, J.M. Lattimer, Phys. Lett. B 531, 167 (2002). DOI 10.1016/S0370-2693(01)01514-3

17. A. Rabhi, C. Providência, J.D. Providência, Journal of Physics G: Nuclear and Particle Physics 35(12), 125201 (2008)

18. D. Lai, S.L. Shapiro, ApJ 383, 745 (1991). DOI 10.1086/ 170831

19. M. Bocquet, S. Bonazzola, E. Gourgoulhon, J. Novak, Astron. Astrophys. 301, 757 (1995)

20. C.Y. Cardall, M. Prakash, J.M. Lattimer, Astrophys. J. 554, 322 (2001). DOI 10.1086/321370

21. D. Chatterjee, T. Elghozi, J. Novak, M. Oertel, Mon. Not. Roy. Astron. Soc. 447, 3785 (2015). DOI 10.1093/mnras/ stu2706

22. N. Chamel, R.L. Pavlov, L.M. Mihailov, C.J. Velchev, Z.K. Stoyanov, Y.D. Mutafchieva, M.D. Ivanovich, J.M. Pearson, S. Goriely, Phys. Rev. C 86, 055804 (2012). DOI 10.1103/PhysRevC.86.055804

23. N. Chamel, Z.K. Stoyanov, L.M. Mihailov, Y.D. Mutafchieva, R.L. Pavlov, C.J. Velchev, Phys. Rev. C 91(6), 065801 (2015). DOI 10.1103/PhysRevC.91.065801 
24. D. Blaschke, N. Chamel, Astrophys. Space Sci. Libr. 457, 337 (2018). DOI 10.1007/978-3-319-97616-7_7

25. R.C.R. de Lima, S.S. Avancini, C. Providência, Phys. Rev. C 88, 035804 (2013)

26. S.S. Bao, J.N. Hu, H. Shen, Phys. Rev. C 103(1), 015804 (2021). DOI 10.1103/PhysRevC.103.015804

27. H. Pais, B. Bertolino, J. Fang, X. Wang, C. Providência, (2021)

28. I. Sengo, H. Pais, B. Franzon, C. Providência, Phys. Rev. D 102(6), 063013 (2020). DOI 10.1103/PhysRevD.102. 063013

29. D.G. Yakovlev, C.J. Pethick, Ann. Rev. Astron. Astrophys. 42, 169 (2004). DOI 10.1146/annurev.astro.42. 053102.134013

30. D. Viganò, N. Rea, J.A. Pons, R. Perna, D.N. Aguilera, J.A. Miralles, Mon. Not. Roy. Astron. Soc. 434, 123 (2013). DOI 10.1093/mnras/stt1008

31. A.Y. Potekhin, D.G. Yakovlev, Astron. Astrophys. 374, 213 (2001). DOI 10.1051/0004-6361:20010698

32. J.A. Pons, J.A. Miralles, U. Geppert, Astron. Astrophys. 496(1), 207 (2009). DOI 10.1051/0004-6361:200811229

33. A.Y. Potekhin, G. Chabrier, Astron. Astrophys. 609, A74 (2018). DOI 10.1051/0004-6361/201731866

34. T. Akgün, P. Cerdá-Durán, J.A. Miralles, J.A. Pons, Mon. Not. Roy. Astron. Soc. 481(4), 5331 (2018). DOI 10.1093/ mnras/sty2669

35. A.M. Beloborodov, X. Li, Astrophys. J. 833(2), 261 (2016). DOI 10.3847/1538-4357/833/2/261

36. M. Arnould, S. Goriely, K. Takahashi, Physics Reports 450(4-6), 97-213 (2007). DOI 10.1016/j.physrep.2007. 06.002. URL http://dx.doi.org/10.1016/j.physrep. 2007.06 .002

37. S. Goriely, N. Chamel, H.T. Janka, J.M. Pearson, Astron. Astrophys. 531, A78 (2011). DOI 10.1051/0004-6361/ 201116897

38. T. Carreau, F. Gulminelli, N. Chamel, A.F. Fantina, J.M. Pearson, Astron. Astrophys. 635, A84 (2020). DOI 10. 1051/0004-6361/201937236

39. A.Y. Potekhin, G. Chabrier, Phys. Rev. E 62, 8554 (2000). DOI 10.1103/PhysRevE.62.8554. URL https://link. aps.org/doi/10.1103/PhysRevE.62.8554

40. C.J. Pethick, A.Y. Potekhin, Phys. Lett. B 427, 7 (1998). DOI 10.1016/S0370-2693(98)00341-4

41. G. Watanabe, K. Iida, K. Sato, Nucl. Phys. A 687, 512 (2001). DOI 10.1016/S0375-9474(00)00585-6

42. G. Watanabe, K. Iida, K. Sato, Nuclear Physics A 726(3), 357 (2003). DOI https://doi.org/10.1016/S0375-9474(03) 01601-4. URL https://www.sciencedirect.com/ science/article/pii/S0375947403016014

43. A. Rabhi, C. Providência, J.D. Providência, Journal of Physics G: Nuclear and Particle Physics 35(12), 125201 (2008)

44. C.J. Horowitz, J. Piekarewicz, Physical Review Letters 86(25), 5647 (2001)

45. C. Providencia, A. Rabhi, Phys. Rev. C 87(5), 055801 (2013). DOI 10.1103/PhysRevC.87.055801

46. H. Pais, C. Providência, Phys. Rev. C94(1), 015808 (2016)

47. Y. Sugahara, H. Toki, Nucl. Phys. A 579, 557 (1994). DOI 10.1016/0375-9474(94)90923-7

48. S.S. Bao, J.N. Hu, Z.W. Zhang, H. Shen, Phys. Rev. C 90(4), 045802 (2014). DOI 10.1103/PhysRevC.90.045802

49. M. Fortin, C. Providencia, A.R. Raduta, F. Gulminelli, J.L. Zdunik, P. Haensel, M. Bejger, Phys. Rev. C 94(3), 035804 (2016). DOI 10.1103/PhysRevC.94.035804
50. H. Shen, F. Ji, J. Hu, K. Sumiyoshi, Astrophys. J. 891 , 148 (2020). DOI 10.3847/1538-4357/ab72fd

51. K. Hebeler, J.M. Lattimer, C.J. Pethick, A. Schwenk, Astrophys. J. 773, 11 (2013). DOI 10.1088/0004-637X/773/ $1 / 11$

52. D. Adhikari, et al., Phys. Rev. Lett. 126(17), 172502 (2021). DOI 10.1103/PhysRevLett.126.172502

53. B.T. Reed, F.J. Fattoyev, C.J. Horowitz, J. Piekarewicz, (2021)

54. L. Brito, C. Providência, A.M. Santos, S.S. Avancini, D. Menezes, P. Chomaz, Phys. Rev. C 74, 045801 (2006)

55. H. Pais, A. Santos, L. Brito, C. Providência, Phys. Rev. C 82, 025801 (2010)

56. H. Müller, B.D. Serot, Phys. Rev. C 52, 2072 (1995)

57. V. Baran, M. Colonna, M.D. Toro, A.B. Larionov, Nucl. Phys. A 632, 287 (1998)

58. J. Margueron, P. Chomaz, Phys. Rev. C 67, 041602(R) (2003)

59. J. Frieben, L. Rezzolla, Mon. Not. Roy. Astron. Soc. 427, 3406 (2012). DOI 10.1111/j.1365-2966.2012.22027.x

60. S.K. Lander, P. Haensel, B. Haskell, J.L. Zdunik, M. Fortin, Mon. Not. Roy. Astron. Soc. 503(1), 875 (2021). DOI 10.1093/mnras/stab460

61. K. Uryu, S. Yoshida, E. Gourgoulhon, C. Markakis, K. Fujisawa, A. Tsokaros, K. Taniguchi, Y. Eriguchi, Phys. Rev. D 100(12), 123019 (2019). DOI 10.1103/PhysRevD.100. 123019

62. C. Ducoin, J. Margueron, C. Providência, I. Vidana, Phys. Rev. C 83, 045810 (2011)

63. K. Uryu, E. Gourgoulhon, C. Markakis, K. Fujisawa, A. Tsokaros, Y. Eriguchi, Phys. Rev. D 90(10), 101501 (2014). DOI 10.1103/PhysRevD.90.101501

64. S.K. Lander, N. Andersson, D. Antonopoulou, A.L. Watts, Mon. Not. Roy. Astron. Soc. 449(2), 2047 (2015). DOI $10.1093 / \mathrm{mnras} / \mathrm{stv} 432$

65. S.K. Lander, K.N. Gourgouliatos, Mon. Not. Roy. Astron. Soc. 486(3), 4130 (2019). DOI 10.1093/mnras/stz1042 